of 6 hours per day and had availed no more than 15 days of leave in the last 6 months.

Result The mean age of the PTs was 29.5 years. On an average, the PTs worked for $8 \pm 1.2$ hours per day for 6 days a week. $78 \%$ of the PTs complained of pain or discomfort within the past 6 months. The commonest sites of pain were lower back (58\%), neck (52\%), upper back (50\%), wrist and hand $(35 \%)$, shoulder (32\%) and ankle (10\%). Analysis of short form of workstyle questionnaire revealed that $70 \%$ of the subjects reported an adverse workstyle risk (total score $>28$ ). The perceived exertion and workload were also high as over $75 \%$ of the PTs had scores of $>15$ (Borg CR 10) and $>50$ (NASA Task Load Index) respectively.

Discussion PTs handling both adult and paediatric patients had high risk of developing WRMSD and appropriate recommendations were given based on the results to ensure prevention of WRMSD.

\section{EFFECTIVENESS OF ONSITE OCCUPATIONAL HEALTH CLINICS IN MANAGEMENT OF WORK RELATED MUSCULOSKELETAL DISORDERS IN 10,850 INFORMATION TECHNOLOGY PROFESSIONALS}

\begin{abstract}
${ }^{1}$ Deepak Sharan*, ${ }^{2}$ Joshua Samuel Rajkumar, ${ }^{2}$ Rajarajeshwari Balakrishnan, ${ }^{3}$ Jerrish A Jose. ${ }^{1}$ Department of Orthopaedics, RECOUP Neuromusculoskeletal Rehabilitation Centre, Bangalore, India; ${ }^{2}$ Research and Development, RECOUP Neuromusculoskeletal Rehabilitation Centre, Bangalore, India; ${ }^{3}$ Corporate Services, RECOUP Neuromusculoskeletal Rehabilitation Centre, Bangalore, India
\end{abstract}

\subsection{6/oemed-2018-ICOHabstracts.420}

Introduction Onsite clinics play a crucial role in the provision of occupational health services. However, the effectiveness of onsite clinics in the management of work related musculoskeletal disorders (WRMSD) is an under studied area. This study reports the outcome of onsite occupational health clinics in Information Technology (IT) companies in the management of WRMSD over a 10 year period.

Methods Aprospective study was conducted from 2006 to 2016, covering 10850 employees of IT companies in different cities in an Industrially Developing Country. The employees (6990 males and 3860 females, between the ages 20 to 60 years), were diagnosed by an experienced occupational health physician (OHP) to have a WRMSD in specific regionsfollowing extensive usage of desktop and/or laptop computer. All the employees then underwent an ergonomic workplace analysis and protocol based rehabilitation for the WRMSD by specially trained occupational physiotherapists. The employees were reviewed by the OHP monthly and at the completion of rehabilitation.

Result Most employees were software and application engineers, followed by managers and technical support staff. A total of $62 \%$ of the employees worked for at least 5-9 hours per day and 38\% for 10-14 hours per day. The predominant symptoms were low back pain (for males) andneck pain (for females). Nearly, 55\% were diagnosed to haveMyofascial Pain Syndrome and others with Thoracic Outlet Syndrome, Fibromyalgia, Tendinopathies and Type 1 Complex Regional Pain Syndrome. After the rehabilitation, the VAS scale showed significant reduction in pain levels $(\mathrm{p}<0.01) .78 \%$ had reported reduced productivity due to the WRMSD, which improved markedly after the rehabilitation. 93\% of workers reported complete resolution of symptoms and $7 \%$ reported partial resolution of symptoms but could work without restriction.
Discussion Onsite occupational health clinics are effective in the managementof WRMSD in IT companies.

\section{WORK RELATED MUSCULOSKELETAL DISORDERS AMONG ORTHOPAEDIC SURGEONS: A SURVEY STUDY}

${ }^{1}$ Deepak Sharan*, ${ }^{2}$ Joshua Samuel Rajkumar, ${ }^{2}$ Rajarajeshwari Balakrishnan. ${ }^{1}$ Department of Orthopaedics, RECOUP Neuromusculoskeletal Rehabilitation Centre, Bangalore, India; ${ }^{2}$ Research and Development, RECOUP Neuromusculoskeletal Rehabilitation Centre, Bangalore, India

\subsection{6/oemed-2018-ICOHabstracts.421}

Introduction Surgeons, especially Orthopaedic Surgeons (OS) maintain awkward postures and repetitive tasks which are ergonomically risky. However, there is a paucity of data on the prevalence of work related musculoskeletal disorders (WRMSD) among OS. Hence, the objective was to evaluate the prevalence and risk factors of WRMSD among OS.

Methods A survey was conducted using a structured questionnaire, disseminated online. There were 57 respondents, who were OS with a minimum working experience of one year, and the surgeons were practicing in the field of orthopaedics only. The structured questionnaire included demographic details such as age, sex, height, weight, total work experience, number of working hours in a day, type or department of work, questions related to regular exercise, physical risk factors associated with working condition, present health status. Nordic Musculoskeletal Questionnaire (NMQ) was used to know the regional involvement, prevalence and disability rate of MSD, in past 7 days or during the last 12 months. The short form of work style questionnaire was used to assess the risk factors of adverse work style. Data were recorded and analysed.

Result The mean age of the OSs was 46.32 years and were predominantly males (96\%). On an average, the percentage of OSs with operating hours more than 14 hours was $75.4 \%$. Joint replacement (50.9\%) and Arthroscopic surgeries (35.1\%), were the commonest surgical procedures carried out by them. A high prevalence rate of work related musculoskeletal symptoms among OS was found, mainly in the low back (68.42\%), neck (56.14\%), shoulder (42.1\%) and upper back (31.57\%) regions. Sustained static and/or awkward posture was perceived as the factor most commonly associated with low back and neck symptoms by $84.2 \%$ of respondents.

Discussion A high prevalence of musculoskeletal symptoms was reported among OS and interventions to address the risk factors identified are recommended.

\section{RISK FACTORS FOR THE DEVELOPMENT OF WORK RELATED MUSCULOSKELETAL DISORDERS AMONG INFORMATION TECHNOLOGY PROFESSIONALS}

'Deepak Sharan*, ${ }^{2} J$ oshua Samuel Rajkumar. 'Department of Orthopaedics, RECOUP Neuromusculoskeletal Rehabilitation Centre, Bangalore, India; ${ }^{2}$ Research and Development, RECOUP Neuromusculoskeletal Rehabilitation Centre, Bangalore, India

10.1136/oemed-2018-ICOHabstracts.422

Introduction Work Related Musculoskeletal Disorders (WRMSD) are highly prevalent among Information Technology (IT) professionals. However, the risk factors associated with the development of WRMSD are not clear. Hence, the 